\title{
CONVOLUTION EQUATIONS FOR VECTOR-VALUED ENTIRE FUNCTIONS OF NUCLEAR BOUNDED TYPE
}

\author{
BY
}

THOMAS A. W. DWYER III

\begin{abstract}
Given two complex Banach spaces $E$ and $F$, convolution operators "with scalar coefficients" are characterized among all convolution operators on the space $H_{N b}\left(E^{\prime} ; F\right)$ of entire mappings of bounded nuclear type of $E^{\prime}$ into $F$. The transposes of such operators are characterized as multiplication operators in the space $\operatorname{Exp}\left(E ; F^{\prime}\right)$ of entire mappings of exponential type of $E$ into $F^{\prime}$. The division theorem for entire functions of exponential type of Malgrange and Gupta is then extended to the case when one factor is vectorvalued. With this tool the following "vector-valued" existence and approximation theorems for convolution equations are proved: THEOREM 1. Nonzero convolution operators "of scalar type" are surjective on $H_{N b}\left(E^{\prime} ; F\right)$.

THEOREM 2. Solutions of homogeneous convolution equations of scalar type can be approximated in $H_{N b}\left(E^{\prime} ; F\right)$ by exponential-polynomial solutions.
\end{abstract}

Introduction. The theory of existence and approximation of solutions of linear partial differential equations and convolution equations in infinite-dimensional domains has been developed by Ph. Boland [B1-3], S. Dineen [Di], Boland with Dineen [B-D], T. Dwyer [D1-6], C. Gupta [G1-3], M. Matos [Mat], Gupta with Nachbin [N2] and D. Pisanelli [P] (the latter dealing rather with total differential equations).

Other than $[\mathbf{P}]$, which does not deal with convolutions or partial differential equations, the results in the references above are for scalar-valued functions.

Counterexamples found by Aron and Boland [A-B] indicate that not all convolution equations have solutions among vector-valued functions, even in finite dimension. The purpose of this paper is to show that, if one restricts oneself to convolution operators of "scalar type" (see Chapter II), i.e., which reduce for finite-dimensional domains to linear differential operators (of possibly infinite order) with scalar coefficients, then one recovers the Malgrange-Gupta existence and approximation theorems for "nuclear" entire functions with domain and values in Banach spaces.

Received by the editors June 4, 1974 and, in revised form, September 25, 1974.

AMS (MOS) subject classifications (1970). Primary 35E99, 46G2 0, 58C20; Secondary 46E10, 32A15.

Key words and phrases. Infinite-dimensional holomorphy, convolution equations, vector-valued entire functions. 
This paper is organized as follows:

In Chapter I we establish the necessary duality between the space $P_{N}\left({ }^{n} E^{\prime} ; F\right)$ of $n$-homogeneous polynomials from a dual Banach space $E^{\prime}$ onto a Banach space $F$, and the space $P\left({ }^{n} E ; F^{\prime}\right)$ of all continuous $n$-homogeneous polynomials from $E$ into $F^{\prime}$. This duality is described by a bilinear form $\langle,\rangle_{n, F}$ and the chapter ends with a formula that allows the representation of $\langle,\rangle_{n, F}$ in terms of the analogous form $\langle,\rangle_{n}$ between scalar-valued polynomials.

In Chapter II we develop in turn the duality between the space $H_{N b}\left(E^{\prime} ; F\right)$ of entire functions of nuclear bounded type from $E^{\prime}$ into $F$, and the space $\operatorname{Exp}\left(E ; F^{\prime}\right)$ of entire functions of exponential type from $E$ into $F^{\prime}$. This duality is described by a bilinear form $\langle,\rangle_{F}$, and the chapter ends with a formula that allows the representation of $\left\langle\langle,\rangle_{F}\right.$ by the corresponding form $\langle$, $\rangle$ between scalar-valued functions.

In Chapter III the "homogeneous (partial) differential operators of scalar type" $g_{n}^{\prime}(d)_{F}$, associated with homogeneous scalar-valued polynomials $g_{n}^{\prime}$ on $E$, are defined between spaces of vector-valued homogeneous nuclear polynomials on $E^{\prime}$, by extension of the scalar operators $g_{n}^{\prime}(d)$, using the fact that $P_{N}\left({ }^{n} E^{\prime} ; F\right)$ is the nuclear completion of $P_{N}\left({ }^{n} E^{\prime}\right) \otimes F$. These operators are then extended to all of $H_{N b}\left(E^{\prime} ; F\right)$ in the way used by Dineen to treat differential operators on formal power series [Di, Chapter 3]. "Scalar differential operators of infinite order" $g^{\prime}(d)_{F}$, associated with scalar-valued entire functions of exponential type $\boldsymbol{g}^{\prime}$ on $E$, are then constructed by power series expansions, and are shown to be the "convolution operators" $\vec{T}^{*}$, with $\vec{T}=T \otimes 1_{F}$ in $L\left(H_{N b}\left(E^{\prime} ; F\right) ; F\right)$, where $T \in H_{N b}\left(E^{\prime}\right)^{\prime}, 1_{F}$ is the identity operator on $F$, and

$$
(\vec{T} * \vec{f})\left(x^{\prime}\right)=\vec{T}\left(u^{\prime} \mapsto \vec{f}\left(x^{\prime}+u^{\prime}\right)\right) .
$$

The chapter ends with a proof that the adjoint of $g^{\prime}(d)_{F}$ with respect to the pairing $\langle l,\rangle_{F}$ is the operator $g^{\prime} \cdot$ of pointwise multiplication by $g^{\prime}$.

In Chapter IV Gupta's fundamental division theorem for scalar-valued functions of exponential type is rewritten in the formalism of the bilinear form 《, , , and is then extended to the case when one of the factors is vector-valued. The chapter ends with proofs of the existence theorem $\left(g^{\prime}(d)_{F}\right.$ is surjective on $H_{N b}\left(E^{\prime} ; F\right)$ ) and the approximation theorem (exponential-polynomial solutions are total among the solutions $\vec{f} \in H_{N b}\left(E^{\prime} ; F\right)$ of $\left.g^{\prime}(d)_{F} \vec{f}=0\right)$.

$A$ list of references is provided at the end. In particular, an excellent exposition of the theory of convolution equations on $\mathrm{H}_{N b}\left(E^{\prime}\right)$ (the scalar-valued predecessor of this paper), as well as the more general "unbounded" case of $H_{N}\left(E^{\prime}\right)$, is found in [G2]. A similar study of operators on $H_{N}\left(E^{\prime} ; F\right)$ can presumably be carried out by the use of the density of scalar-valued functions in $H_{N}\left(E^{\prime} ; F\right)$, proved by Aron in [A]. 


\section{Chapter I. Vector-Valued Polynomials}

Definitions AND NOtATion. In all that follows $E$ and $F$ are complex Banach spaces (for simplicity with the approximation property, in order to have a one-to-one correspondence between nuclear tensors and nuclear polynomials, although this can be bypassed (see below)). $E^{\prime}, F^{\prime}$ are the Banach space duals, and $E^{*}, F^{*}$ the vector space duals, of $E$ and $F$ respectively. Given $x \in E$ and $x^{\prime} \in E^{\prime}$ (or $E^{*}$ ) we use the notation $\left\langle x, x^{\prime}\right\rangle:=x^{\prime}(x)$, and similarly for the natural pairing of $F$ with $F^{\prime}$. Notation is as in [N1] and [G2]: $P\left({ }^{n} E^{\prime} ; F\right)$ is the Banach space of all continuous $n$-homogeneous polynomials from $E^{\prime}$ to $F$. The space $P\left({ }^{n} E ; F^{\prime}\right)$ of continuous $n$-homogeneous polynomials $P_{n}^{\prime}$ from $E$ to $F^{\prime}$ is similarly defined.

$P_{N}\left({ }^{n} E^{\prime} ; F\right)$ is the Banach space of $n$-homogeneous nuclear polynomials from $E^{\prime}$ to $F$, induced from the nuclear tensor product $E \otimes \cdots \otimes E \otimes F$ (not $E^{\prime \prime}$ ). $P_{f}\left({ }^{n} E^{\prime} ; F\right)$ is the dense subspace of polynomials of the form $\Sigma_{i}\left\langle u_{i},\right\rangle^{n} v_{i}$, with $u_{i} \in E$ and $v \in F$.

If $\operatorname{dim} F=1$ we write $P\left({ }^{n} E^{\prime}\right)$ for $P\left({ }^{n} E^{\prime} ; F\right)$ and similarly for all other spaces of polynomials. Polynomials are then written as $P$ or $P^{\prime}$ in place of $\vec{P}$ or $\vec{P}^{\prime}$.

DuAlity OF SPACES OF HOMOGENEOUS POLYNOMIALS. Given $T \in$ $P_{N}\left({ }^{n} E^{\prime} ; F\right)^{\prime}$ let $J_{n} T: E \rightarrow F^{*}$ be defined by $\left\langle y, J_{n} T(x)\right\rangle:=\left\langle x^{n} \cdot y, T\right\rangle$ for all $x \in E$ and $y \in F$. We have:

Proposition I.1. The mapping $T \mapsto J_{n} T$ is an isometry from $P_{N}\left({ }^{n} E^{\prime} ; F\right)^{\prime}$ onto $\mathrm{P}\left({ }^{n} E ; F^{\prime}\right)$.

Proof. Given $T \in \cdot P_{N}\left({ }^{n} E^{\prime} ; F\right)^{\prime}$ we have $J_{n} T(E) \subset F^{\prime}:$ indeed,

$$
\left\langle y, J_{n} T(x)\right\rangle|=|\left\langle x^{n} \cdot y, T\right\rangle \mid \leqslant\left\|x^{n} \cdot y\right\|_{N}\|T\|=\|x\|^{n}\|y\|\|T\|,
$$

so $\left\|J_{n} T(x)\right\| \leqslant\|x\|^{n}\|T\|$, for all $x \in E$. From this point the proof is the same as in the scalar-valued case [G2, §7, Lemma 4, p. 59].

Definition. The duality between $P_{N}\left({ }^{n} E^{\prime} ; F\right)$ and $P\left({ }^{n} E ; F^{\prime}\right)$ can also be described by means of the bilinear form

$$
\langle,\rangle_{n, F}: P_{N}\left({ }^{n} E^{\prime} ; F\right) \times P\left({ }^{n} E ; F^{\prime}\right) \rightarrow C
$$

defined by $\left\langle\vec{P}_{n}, \vec{P}_{n}^{\prime}\right\rangle_{n, F}:=\left\langle\vec{P}_{n}, T_{n}\right\rangle$, where $T_{n}:=J_{n}^{-1} \vec{P}_{n}^{\prime}$. It follows that $\left\langle\vec{P}_{n}, \vec{P}_{n}^{\prime}\right\rangle_{n, F} \mid \leqslant\left\|\vec{P}_{n}\right\|_{N}\left\|\vec{P}_{n}^{\prime}\right\|$ for every $\vec{P}_{n} \in P_{N}\left({ }^{n} E^{\prime} ; F\right)$ and $\vec{P}^{\prime} \in P\left({ }^{n} E ; F^{\prime}\right)$. In particular we have $\left\langle x^{n} \cdot y, \vec{P}_{n}^{\prime}\right\rangle_{n, F}=\left\langle y, \vec{P}_{n}^{\prime}(x)\right\rangle$ for all $x \in E$ and $y \in F$.

Let $\langle,\rangle_{n}: P_{N}\left({ }^{n} E^{\prime}\right) \times P\left({ }^{n} E\right) \rightarrow C$ be the bilinear form corresponding to the isometry $P_{N}\left({ }^{n} E^{\prime}\right)^{\prime} \rightarrow P\left({ }^{n} E\right)$ when $\operatorname{dim} F=1$, i.e., $\left\langle x^{n}, P_{n}^{\prime}\right\rangle_{n}=P_{n}^{\prime}(x)$ for all $x \in E$ and $P_{n}^{\prime} \in P\left({ }^{n} E\right)$.

Given $\vec{P}_{n}^{\prime} \in P\left({ }^{n} E ; F^{\prime}\right)$ and $y \in F$, we will use the notation $\vec{P}_{n y}^{\prime}: E \rightarrow C$ to describe the associated scalar-valued polynomial, defined by $\vec{P}_{n y}^{\prime}(x):=\left\langle y, \vec{P}_{n}^{\prime}(x)\right\rangle$. 
Conversely, given $P_{n} \in P_{N}\left({ }^{n} E^{\prime}\right)$ and $y \in F$, the associated vector-valued polynomial $P_{n} \cdot y: E^{\prime} \rightarrow F$ is defined by $\left(P_{n} \cdot y\right)\left(x^{\prime}\right):=P_{n}\left(x^{\prime}\right) y$ for all $x^{\prime} \in E^{\prime}$. Clearly $\vec{P}_{n y}^{\prime} \in P\left({ }^{n} E\right)$ and $P_{n} \cdot y \in P_{N}\left({ }^{n} E^{\prime} ; F\right)$. We will need the following "reduction from $\langle,\rangle_{n, F}$ to $\langle,\rangle_{n} "$ :

Proposition 1.2. $\left\langle P_{n} \cdot y, \vec{P}_{n}^{\prime}\right\rangle_{n, F}=\left\langle P_{n}, \vec{P}_{n y}^{\prime}\right\rangle_{n}$ for all $P_{n} \in P_{N}\left({ }^{n} E^{\prime}\right)$, $\vec{P}_{n}^{\prime} \in P\left({ }^{n} E ; F^{\prime}\right)$ and $y \in F$.

Proof. Since $\langle,\rangle_{n, F}$ and $\langle,\rangle_{n}$ are continuous bilinear forms and $P_{f}\left({ }^{n} E^{\prime}\right)$ is dense in $P_{N}\left({ }^{n} E^{\prime}\right)$ it is enough to consider $P_{n}$ of the form $P_{n}=x^{n}, x \in E$.

\section{Chapter II. Vector-Valued Entire Functions}

DEFINITIONS AND NOTATION. $H\left(E^{\prime} ; F\right)$ is the vector space of entire functions $E^{\prime} \rightarrow F$ (i.e., functions with locally uniform power series expansions $\Sigma_{n}(1 / n !) \hat{d}^{n} \vec{f}\left(x_{0}^{\prime}\right)$ at every $x_{0}^{\prime} \in E^{\prime}$, where $\hat{d}^{n}$ denotes the $n$th Fréchet derivative polynomial. $H_{b}\left(E^{\prime} ; F\right)$ is the subspace of functions bounded on bounded sets, or equivalently with infinite radius of convergence at one or any point $[\mathrm{N1}, \S 7$, Proposition 2]. The spaces $H\left(E ; F^{\prime}\right)$ and $H_{b}\left(E ; F^{\prime}\right)$ are similarly defined. Also $\operatorname{Exp}\left(E ; F^{\prime}\right)$ is the space of functions $\vec{f}^{\prime}=\Sigma_{n=0}^{\infty}(1 / n !) \vec{P}_{n}^{\prime}$ with $\vec{P}_{n}^{\prime} \in P\left({ }^{n} E ; F^{\prime}\right)$ such that lim $\sup _{n}\left\|\vec{P}_{n}^{\prime}\right\|^{1 / n}<\infty$, called entire functions of exponential type from $E$ to $F^{\prime}$, or equivalently, such that $\left\|\vec{f}^{\prime}(x)\right\| \leqslant C e^{c\|x\|}$ for $C \geqslant 1, c \geqslant 0$ and all $x \in E$ (the equivalence follows from the Cauchy estimates [Gं2, §3, Proposition 6, p. 24]). By $H_{N b}\left(E^{\prime} ; F\right)$ we mean, as in [G1], [G2], the Fréchet space of functions $\vec{f}=\Sigma_{n=0}^{\infty}(1 / n !) \vec{P}_{n}$ with $\vec{P}_{n} \in P_{N}\left({ }^{n} E^{\prime} ; F\right)$ such that $\lim _{n}\left\{1 / n !\left\|\vec{P}_{n}\right\|_{N}\right\}^{1 / n}=0$, or equivalently such that $\|\vec{f}\|_{N, \rho}:=\sum_{n=0}^{\infty} \rho^{n} 1 / n !\left\|\vec{P}_{n}\right\|_{N}<\infty$ for all $\rho>0$, equipped with the norms \|\|$_{N, \rho}$ thus defined. These are the entire functions of nuclear bounded type from $E^{\prime}$ to $F$. When $\operatorname{dim} F=1$ we use the notation $H\left(E^{\prime}\right)$ for $H\left(E^{\prime} ; F\right)$, and similarly for the other function spaces. Functions on $E^{\prime}$ are then written as $f$ and functions on $E$ as $f^{\prime}$, instead of $\vec{f}$ and $\vec{f}^{\prime}$.

By [G2, $\$ 5$, Lemma 1, p. 45], Taylor series converge to the corresponding functions in $H_{N b}\left(E^{\prime} ; F\right)$. Hence the nuclear polynomials form a dense subspace. Moreover, by [G2, §5, Proposition 3, p. 45], the functions $e^{x} \cdot y: E^{\prime} \rightarrow F$, where $\left(e^{x} \cdot y\right)\left(x^{\prime}\right):=e^{\left(x, x^{\prime}\right\rangle} y$ for all $x^{\prime} \in E^{\prime}$ and all $x \in E$ and $y \in F$, are total in $H_{N b}\left(E^{\prime} ; F\right)$.

By $\left[G 2, \S 7\right.$, Lemma 1, p. 52] we know that $H_{N b}\left(E^{\prime} ; F\right)$ is stable under translations, i.e., if $\vec{f} \in H_{N b}\left(E^{\prime} ; F\right)$ then $\tau_{x^{\prime}} \vec{f} \in H_{N b}\left(E^{\prime} ; F\right)$ for all $x^{\prime} \in E^{\prime}$, where $\tau_{x^{\prime}}, \vec{f}\left(u^{\prime}\right):=\vec{f}\left(u^{\prime}-x^{\prime}\right)$.

Proposition II.1. $H_{N b}\left(E^{\prime}\right) \otimes F$ is dense in $H_{N b}\left(E^{\prime} ; F\right)$.

Proof. Given $\vec{f} \in H_{N b}\left(E^{\prime} ; F\right)$ and a neighborhood $N$ of $\vec{f}$ in $H_{N b}\left(E^{\prime} ; F\right)$, from the convergence to $\vec{f}$ in $H_{N b}\left(E^{\prime} ; F\right)$ of the Taylor series of $\vec{f}$ it follows that 
there is a polynomial $\Sigma_{n=0}^{N} \vec{P}_{n}$ in $N$ (i.e., $P_{n}=(1 / n !) \hat{d}^{n} f(0)$. Since $P_{N}\left({ }^{n} E^{\prime}\right) \otimes F$ is dense in $P_{N}\left({ }^{n} E^{\prime} ; F\right)$ by construction, it follows that there is a sequence $\left(\vec{Q}_{n, k}\right)_{k}$ of $n$-homogeneous polynomials $\vec{Q}_{n, k} \in P_{N}\left({ }^{n} E^{\prime}\right) \otimes F$ (of finite-dimensional range) converging in $P_{N}\left({ }^{n} E^{\prime} ; F\right)$ to $\vec{P}_{n}$, for each $n \leqslant N$. Since the identity imbeddings $P_{N}\left({ }^{n} E^{\prime} ; F\right) \subset H_{N b}\left(E^{\prime} ; F\right)$ are continuous, it follows that $\left(\vec{Q}_{n, k}\right)_{k}$ converges to $\vec{P}_{n}$ in $H_{N b}\left(E^{\prime} ; F\right)$ for each $n \leqslant N$. Hence $\left(\sum_{n=0}^{N} \vec{Q}_{n, k}\right)_{k}$ converges to $\sum_{n=0}^{N} \vec{P}_{n}$ in $H_{N b}\left(E^{\prime} ; F\right)$, so $\Sigma_{n=0}^{N} Q_{n, k}$ is in $N$ for a sufficiently large $k$. Since clearly $\Sigma_{n=0}^{N} \vec{Q}_{n, k} \in P_{N}\left(E^{\prime}\right) \otimes F \subset H_{N b}\left(E^{\prime}\right) \otimes F$ we are done.

REMARK. A similar (but much deeper) density result that leads to the treatment of the "unbounded" type of $[\mathbf{G 2}, \S 9]$ is given in [A] .

DuAlty OF SPACES OF ENTIRE FUnCtions. Given $T \in H_{N b}\left(E^{\prime} ; F\right)^{\prime}$ let $B T: E \rightarrow F^{*}$ be defined by $\langle y, B T(x)\rangle:=\left\langle e^{x} \cdot y, T\right\rangle$ for all $x \in E$ and $y \in F$. $B T$ is the Fourier-Borel transform of $T$. We have:

Proposition II.2. The mapping $T \mapsto B T$ is a linear isomorphism of $H_{N b}\left(E^{\prime} ; F\right)^{\prime}$ onto $\operatorname{Exp}\left(E ; F^{\prime}\right)$.

Proof. Given $T \in H_{N b}\left(E^{\prime} ; F\right)^{\prime}$ we have $B T(E) \subset F^{\prime}$ : indeed, $|\langle y, T(x)\rangle|$ $:=\left\langle e^{x} \cdot y, T\right\rangle \mid ; T \in H_{N b}\left(E^{\prime} ; F\right)^{\prime}$ implies there is some $\rho>0$, and a constant $\|T\|_{\rho} \geqslant 0$, such that $\left|\left\langle e^{x} \cdot y, T\right\rangle\right| \leqslant\|T\|_{\rho}\left\|e^{x} \cdot y\right\|_{N, \rho} ;$ but

$$
\begin{aligned}
\left\|e^{x} \cdot y\right\|_{N, \rho} & :=\sum_{n=0}^{\infty} \frac{1}{n !} \rho^{n}\left\|x^{n} \cdot y\right\|_{N} \\
& =\|y\| \sum_{n=0}^{\infty} \frac{1}{n !} \rho^{n}\|x\|^{n}=\|y\| e^{\rho\|x\|,}
\end{aligned}
$$

so $|\langle y, B T(x)\rangle| \leqslant\|T\|_{\rho}\|y\| e^{\rho\|x\|}$, i.e., $\|B T(x)\| \leqslant\|T\|_{\rho} e^{\rho\|x\|}<\infty$.

$B T \in \operatorname{Exp}\left(E ; F^{\prime}\right):$ let $T_{n}:=$ restriction of $T$ to $P_{N}\left({ }^{n} E^{\prime} ; F\right)$ and $P_{n}^{\prime}:=J_{n} T_{n}$ $\in P\left({ }^{n} E ; F^{\prime}\right)$ : as in the proof of the scalar-valued case [G2, $\$ 7$, Proposition 2, p. 60] for each $N$ we get

$$
\begin{aligned}
\left\langle y, \sum_{n=0}^{N} \frac{1}{n !} \vec{P}_{n}^{\prime}(x)\right\rangle & =\sum_{n=0}^{N} \frac{1}{n !}\left\langle y, \vec{P}_{n}^{\prime}(x)\right\rangle \\
& =\sum_{n=0}^{N} \frac{1}{n !}\left\langle x^{n} \cdot y, T_{n}\right\rangle=\left\langle\sum_{n=0}^{N} \frac{1}{n !} x^{n} \cdot y, T\right\rangle,
\end{aligned}
$$

which converges to $\left\langle e^{x} \cdot y, T\right\rangle$, i.e., $\langle y, B T(x)\rangle$, as $N \rightarrow \infty$. That is,

$$
\sum_{n=0}^{N} \frac{1}{n !} \vec{P}_{n}^{\prime}(x) \rightarrow B T(x)
$$

in the weak* topology, for all $x \in E$. Moreover, in the dual norm of $F^{\prime}$ we get $\left\|\Sigma_{n=0}^{N}(1 / n !) \vec{P}_{n}^{\prime}(x)\right\| \leqslant\|T\|_{\rho} e^{\rho\|x\|} \leqslant \infty$ for $\|T\|_{\rho}$ as defined above for some $\rho>0$, so that $\Sigma_{n=0}^{\infty}(1 / n !) \vec{P}^{\prime}(x)$ converges in $F^{\prime}$ (strongly), hence also weakly*, hence 
$\sum_{n=0}^{\infty}(1 / n !) \vec{P}_{n}^{\prime}(x)=B T(x)$ also strongly on $F^{\prime}$. The remainder of the proof is as in the scalar-valued case [G2, §7, Proposition 2].

Definition. The duality between $H_{N b}\left(E^{\prime} ; F\right)$ and $\operatorname{Exp}\left(E ; F^{\prime}\right)$ can also be described by means of the bilinear form $《,\rangle_{F}: H_{N b}\left(E^{\prime} ; F\right) \times \operatorname{Exp}\left(E ; F^{\prime}\right) \rightarrow C$ defined by $\left\langle\left\langle\vec{f}, \vec{f}^{\prime}\right\rangle\right\rangle_{F}:=\langle\vec{f}, T\rangle$, where $T=B^{-1} \vec{f}^{\prime} \in H_{N}\left(E^{\prime} ; F\right)^{\prime}$, for all $\vec{f} \in$ $H_{N b}\left(E^{\prime} ; F\right)$. In particular we have $\left\langle\left\langle e^{x} \cdot y, \vec{f}^{\prime}\right\rangle_{F}=\left\langle y, \vec{f}^{\prime}(x)\right\rangle\right.$ for all $x \in E$ and $y \in F$.

Proposition II.3.

$$
\left.\left\langle\vec{f}, \vec{f}^{\prime}\right\rangle\right\rangle_{F}=\sum_{n=0}^{\infty} \frac{1}{n !}\left\langle\hat{d}^{n} \vec{f}(0), \hat{d}^{n} \vec{f}^{\prime}(0)\right\rangle_{n, F}
$$

for all $\vec{f} \in H_{N b}\left(E^{\prime} ; F\right)$ and $\vec{f}^{\prime} \in \operatorname{Exp}\left(E ; F^{\prime}\right)$.

Proof. Let $\vec{f}^{\prime}=B T, T \in H_{N b}\left(E^{\prime} ; F\right)^{\prime}$, and let $T_{n}:=$ restriction of $T$ to $P_{N}\left({ }^{n} E^{\prime} ; F\right)$ : then $\langle y, B T(x)\rangle:=\left\langle e^{x} \cdot y, T\right\rangle=\Sigma_{n=0}^{\infty}(1 / n !)\left\langle x^{n} \cdot y, T_{n}\right\rangle=$ $\left\langle y, \Sigma_{n=0}^{\infty}(1 / n !) J_{n} T_{n}(x)\right\rangle$, so $\hat{d}^{n} \vec{f}^{\prime}(0)=J_{n} T_{n}$. Hence

$$
\begin{aligned}
\left\langle\left\langle\vec{f}, \vec{f}^{\prime}\right\rangle\right\rangle_{F} & :=\langle\vec{f}, T\rangle=\sum_{n=0}^{\infty} \frac{1}{n !}\left\langle\hat{d}^{n} \vec{f}(0), T\right\rangle=\sum_{n=0}^{\infty} \frac{1}{n !}\left\langle\hat{d}^{n} \vec{f}(0), J_{n} T_{n}\right\rangle_{n, F} \\
& =\sum_{n=0}^{\infty} \frac{1}{n !}\left\langle\hat{d}^{n} \vec{f}(0), \hat{d}^{n} f^{\prime}(0)\right\rangle_{n, F} . \quad \square
\end{aligned}
$$

Let 《 , 》: $H_{N b}\left(E^{\prime}\right) \times \operatorname{Exp}(E) \rightarrow C$ be the bilinear form given by the Fourier-Borel isomorphism when $\operatorname{dim} F=1$, i.e., $\left\langle\left\langle e^{x}, f^{\prime}\right\rangle\right\rangle=f^{\prime}(x)$ for all $f^{\prime} \in$ $\operatorname{Exp}(E)$ and $x \in E$. Clearly Proposition II.3 holds with $《,\rangle_{F}$ replaced by $《, \Downarrow$ and $\langle,\rangle_{n, F}$ by $\langle,\rangle_{n}$.

Given $\vec{f}^{\prime} \in \operatorname{Exp}\left(E ; F^{\prime}\right)$ and $y \in F$, the associated scalar-valued function $\vec{f}_{y}^{\prime}$ : $E \rightarrow C$ is defined by $\vec{f}_{y}^{\prime}(x):=\left\langle y, f^{\prime}(x)\right\rangle$ for every $x \in E$. Conversely, given $f \in$ $H_{N b}\left(E^{\prime}\right)$ the associated vector-valued function $f \cdot y: E^{\prime} \rightarrow F$ is defined by $(f \cdot y)\left(x^{\prime}\right):=f\left(x^{\prime}\right) y$. It is easy to see that $\vec{f}_{y}^{\prime} \in \operatorname{Exp}(E)$ when $\vec{f}^{\prime} \in \operatorname{Exp}\left(E ; F^{\prime}\right)$, and from the equality $\|f \cdot y\|_{N, \rho}=\|f\|_{N, \rho}\|y\|$, easily checked, it follows that $f \cdot y \in H_{N b}\left(E^{\prime} ; F\right)$ when $f \in H_{N b}\left(E^{\prime}\right)$. As for $\langle,\rangle_{n, F}$ and $\langle,\rangle_{n}$ on polynomials, we have the following "reduction of $\langle\text {, }\rangle_{F}$ to $\langle$, $\rangle$ ":

Proposition II.4. $\left\langle f \cdot y, \vec{f}^{\prime}\right\rangle_{F}=\left\langle\left\langle f, \vec{f}_{y}^{\prime}\right\rangle\right.$ for all $f \in H_{N b}\left(E^{\prime}\right), \vec{f}^{\prime} \in$ $\operatorname{Exp}\left(E ; F^{\prime}\right)$ and $y \in F$.

Proof. From the observation that $\hat{d}^{n}(f \cdot y)(0)=\hat{d}^{n} f(0) \cdot y$ and $\hat{d}^{n}\left(\vec{f}_{y}^{\prime}\right)(0)=\hat{d}^{n} \vec{f}^{\prime}(0)_{y}$, the equality above follows from the identities

$$
\left\langle\hat{d}^{n} f(0) \cdot y, \hat{d}^{n} \vec{f}(0)\right\rangle_{n, F}=\left\langle\hat{d}^{n} f(0), \hat{d}^{n} \vec{f}^{\prime}(0)_{y}\right\rangle_{n}
$$

given by Proposition I.2. 


\section{Chapter III. Differential and Convolution Operators}

In $[\mathbf{G 1}, 2]$, differential operators of infinite order, in the form of convolution operators (see ahead) on $H_{N b}\left(E^{\prime}\right)$, are defined and shown to be continuous [G2, §7, Definitions 1 and 2, Proposition 1, pp. 51, 52]. Their natural extensions to $H_{N, b}\left(E^{\prime}\right) \otimes F$ can be obtained by "tensoring" with the identity operator on $F$. However, the proof of the continuity of such extensions with respect to the topology of $\mathrm{H}_{N b}\left(E^{\prime} ; F\right)$ appears to be nontrivial when attempted directly. (If $\operatorname{dim} E<\infty$ then $H_{N b}\left(E^{\prime}\right)=H\left(E^{\prime}\right)$ is nuclear, from which it follows that the nuclear topology on $H_{N b}\left(E^{\prime}\right) \otimes F=H\left(E^{\prime}\right) \otimes F$ coincides with the topology induced by $H_{N b}\left(E^{\prime} ; F\right)=H\left(E^{\prime} ; F\right)[\mathrm{Gr}, \S 7$, Proposition 8, p. 79]. The continuity of the extension of convolution operators to vector-valued functions is then immediate [Tr, Proposition 43.6]. If $\operatorname{dim} E=\infty$, however, then $H_{N b}(E)$ is not nuclear, since $E$ is imbedded in it, by the imbedding of $P_{N}\left({ }^{n} E^{\prime}\right)$ with $n=1$.) Hence we will first define "homogeneous" differential operators acting on homogeneous vector-valued nuclear polynomials (using the fact that $P_{N}\left({ }^{n} E^{\prime} ; F\right)$ is the nuclear norm completion of $P_{N}\left({ }^{n} E^{\prime}\right) \otimes F$ by definition), defining finally the general operators by an "infinite series" of homogeneous operators. (This is in fact the method used by Dineen to define differential operators in terms of homogeneous ones, acting on formal power series (but scalar-valued) in [Di, §3, Definition 13], as well as in [D1], [D2].)

DEFinition. Given $P_{n}^{\prime} \in P\left({ }^{n} E\right)$, corresponding to a $T_{n} \in P_{N}\left({ }^{n} E^{\prime}\right)^{\prime}$ by the isometry $J_{n}$ of Proposition I.1, we define the homogeneous differential operator $P_{n}^{\prime}(d)$ on $Q_{m+n} \in P_{N}\left({ }^{m+n} E^{\prime}\right)$ by $P_{n}^{\prime}(d) Q_{m+n}\left(x^{\prime}\right):=\left\langle\hat{d}^{n} Q_{m+n}\left(x^{\prime}\right), P_{n}^{\prime}\right\rangle_{n}$ $\left(:=\left\langle\hat{d}^{n} Q_{m+n}\left(x^{\prime}\right), T_{n}\right\rangle\right)$ for every $x^{\prime} \in E^{\prime}$.

REMARK. If $P_{n}^{\prime}=u_{1}^{\prime} \cdots u_{n}^{\prime}$ for $u_{1}^{\prime}, \ldots, u_{n}^{\prime}$ in $E^{\prime}$ then $P_{n}^{\prime}(d)$ reduces to $\partial^{n} / \partial u_{1}^{\prime} \cdots \partial u_{n}^{\prime}$ (directional differentiation along the vectors $u_{1}^{\prime}, \ldots, u_{n}^{\prime}$ in $E^{\prime}$ ).

Proposition III.1. If $Q_{m+n} \in P_{N}\left({ }^{m+n} E^{\prime}\right)$ then $P_{n}^{\prime}(d) Q_{m+n} \in P_{N}\left({ }^{m} E^{\prime}\right)$, and has the following properties:

(i) $\left(1 / n\right.$ !) $\left\|P_{n}^{\prime}(d) Q_{m+n}\right\|_{N} \leqslant\left(\begin{array}{c}m+n \\ m\end{array}\right)\left\|P_{n}^{\prime}\right\|\left\|Q_{m+n}^{\prime}\right\|_{N}$;

(ii) $(1 / n !)\left\langle P_{n}^{\prime}(d) Q_{m+n}, Q_{m}^{\prime}\right\rangle_{m}=\left(\begin{array}{c}m+n \\ m\end{array}\right)\left\langle Q_{m+n}, P_{n}^{\prime} \cdot Q_{m}^{\prime}\right\rangle_{m+n}$; for all $Q_{m}^{\prime} \in P\left({ }^{m} E\right)$.

Proof. We first consider polynomials in $P_{f}\left({ }^{m+n} E^{\prime}\right)$, generated by polynomials $Q_{m+n}$ of the form $Q_{m+n}=u^{m+n}, u \in E$. Then

$$
\frac{1}{n !} \hat{d}^{n} Q_{m+n}\left(x^{\prime}\right)=\left(\begin{array}{c}
m+n \\
m
\end{array}\right)\left\langle u, x^{\prime}\right\rangle^{m} u^{n}
$$

so

$$
\frac{1}{n !} P_{n}^{\prime}(d) Q_{m+n}=\left(\begin{array}{c}
m+n \\
m
\end{array}\right) P_{n}^{\prime}(u) u^{m} \in P_{f}\left({ }^{m} E^{\prime}\right)
$$


Then

$$
\begin{array}{r}
\left\langle\frac{1}{n !} P_{n}^{\prime}(d) Q_{m+n}, Q_{m}^{\prime}\right\rangle_{m}=\left(\begin{array}{c}
m+n \\
m
\end{array}\right) P_{n}^{\prime}(u)\left\langle u^{m}, Q_{m}^{\prime}\right\rangle_{m}=\left(\begin{array}{c}
m+n \\
m
\end{array}\right) P_{n}^{\prime}(u) Q_{m}^{\prime}(u) \\
=\left(\begin{array}{c}
m+n \\
m
\end{array}\right)\left\langle u^{m+n}, P_{n}^{\prime} \cdot Q_{m}^{\prime}\right\rangle_{m+n}=\left(\begin{array}{c}
m+n \\
m
\end{array}\right)\left\langle Q_{m+n}, P_{n}^{\prime} \cdot Q_{m}^{\prime}\right\rangle_{m+n},
\end{array}
$$

i.e., (ii) holds for $Q_{m+n} \in P_{f}\left({ }^{m+n} E^{\prime}\right)$. By the Hahn-Banach Theorem there is some $T_{m} \in P_{N}\left({ }^{m} E^{\prime}\right)^{\prime}$ such that $\left\|T_{m}\right\|=1$ and $\left\langle P_{n}^{\prime}(d) Q_{m+n}, T_{m}\right\rangle=\left\|P_{n}^{\prime}(d) Q_{m+n}\right\|_{N}$. Let $Q_{m}^{\prime}$ be the transform $J_{m} T_{m} \in P\left({ }^{m} E\right)$ of $T_{m}$ : then $\left\|Q_{m}^{\prime}\right\|=1$, and by the identity (ii) we have $(1 / n !)\left\|P_{n}^{\prime}(d) Q_{m+n}\right\|_{N}=(1 / n !)\left\langle P_{n}^{\prime}(d) Q_{m+n}, Q_{m}^{\prime}\right\rangle_{m}=$ $\left(\begin{array}{c}m+n \\ m\end{array}\right)\left\langle Q_{m+n}, P_{n}^{\prime} \cdot Q_{m}^{\prime}\right\rangle_{m+n} \leqslant\left(\begin{array}{c}m+n \\ m\end{array}\right)\left\|Q_{m+n}\right\|_{N}\left\|P_{n}^{\prime}\right\|$, i.e., (i) holds on $P_{f}\left({ }^{m+n} E^{\prime}\right)$. Finally, since $P_{f}\left({ }^{m+n} E^{\prime}\right)$ is dense in $P_{N}\left({ }^{m+n} E^{\prime}\right)$, (i) holds for all $Q_{m+n} \in P_{N}\left({ }^{m+n} E^{\prime}\right)$, whence $P_{n}^{\prime}(d)$ is continuous, and since $\left\langle, Q_{m}^{\prime}\right\rangle_{m}$ and $\left\langle, P_{n}^{\prime} \cdot Q_{m}^{\prime}\right\rangle_{m+n}$ are continuous it follows that (ii) also holds for all $Q_{m+n} \in$ $P_{N}\left({ }^{m+n} E^{\prime}\right)$.

DEFINITION. Given $P_{n}^{\prime} \in P\left({ }^{n} E\right)$, the homogeneous differential operator of scalar type $P_{n}^{\prime}(d)_{F}$ is defined on $P_{N}\left({ }^{m+n} E^{\prime}\right) \otimes F$ by $P_{n}^{\prime}(d)_{F}=P_{n}^{\prime}(d) \otimes 1_{F}$, where $1_{F}$ is the identity operator on $F$, i.e., $P_{n}^{\prime}(d)_{F}$ is the linear operator on $P_{N}\left({ }^{m+n} E^{\prime}\right) \otimes F$ derived from the bilinear map

$$
\left(Q_{m+n}, y\right) \in P_{N}\left(m+n E^{\prime}\right) \times F \mapsto P_{n}^{\prime}(d) Q_{m+n} \cdot y \in P_{N}\left(m E^{\prime} ; F\right) \text {. }
$$

Hence $P_{n}^{\prime}(d)_{F}\left(\Sigma_{i} Q_{m+n, i} \cdot y_{i}\right)=\Sigma_{i} P_{n}^{\prime}(d) Q_{m+n, i} \cdot y_{i}$.

Proposition III.1'. Given $P_{n}^{\prime} \in P\left({ }^{n} E\right)$, the operator $P_{n}^{\prime}(d)_{F}$ has a unique continuous linear extension to all $\vec{Q}_{m+n} \in P_{N}\left({ }^{m+n} E^{\prime} ; F\right)$, with following properties:

(i') $(1 / n !)\left\|P_{n}^{\prime}(d)_{F} \vec{Q}_{m+n}\right\|_{N} \leqslant(\underset{m}{m+n})\left\|P_{n}^{\prime}\right\|\left\|\vec{Q}_{m+n}\right\|_{N}$

(ii') $(1 / n !)\left\langle P_{n}^{\prime}(d)_{F} \vec{Q}_{m+n}, \vec{Q}_{m}^{\prime}\right\rangle_{m, F}=\left(\begin{array}{c}m+n \\ m\end{array}\right)\left\langle\vec{Q}_{m+n}, P_{n}^{\prime} \cdot \vec{Q}_{m}\right\rangle_{m+n, F}$; for all $\vec{Q}_{m}^{\prime} \in P\left({ }^{m} E ; F^{\prime}\right)$.

Proof. Let $\vec{Q}_{m+n}=\Sigma_{i} Q_{m+n, i} \cdot y_{i} \in P_{N}\left({ }^{m+n} E^{\prime}\right) \otimes F$, with $Q_{m+n, i} \in$ $P_{N}\left({ }^{m+n} E^{\prime}\right)$ and $y_{i} \in F$ : then from Proposition III.1(i) we get

$$
\begin{aligned}
\frac{1}{n !}\left\|P_{n}^{\prime}(d)_{F} \vec{Q}_{m+n}\right\|_{N} & \leqslant \sum_{i} \frac{1}{n !}\left\|P_{n}^{\prime}(d) Q_{m+n, i}\right\|_{N}\left\|y_{i}\right\| \\
& \leqslant \sum_{i}\left(\begin{array}{c}
m+n \\
m
\end{array}\right)\left\|P_{n}^{\prime}\right\|\left\|Q_{m+n, i}\right\|_{N}\left\|y_{i}\right\| .
\end{aligned}
$$

Since $\left\|\vec{Q}_{m+n}\right\|_{N}=\inf \Sigma_{i}\left\|Q_{m+n, i}\right\|_{N}\left\|y_{i}\right\|$ for all representations $\Sigma_{i} Q_{m+n, i} \cdot y_{i}$ of $\vec{Q}_{m+n}$, the inequality (i') follows. Hence $P_{n}^{\prime}(d)_{F}$ is continuous, so by density of $P_{N}\left({ }^{m+n} E^{\prime}\right) \otimes F$ in $P\left({ }^{m+n} E^{\prime} ; F\right)$ it follows that $P_{n}^{\prime}(d)_{F}$ has a unique continuous linear extension to all $Q_{m+n} \in P_{N}\left({ }^{m+n} E^{\prime} ; F\right)$ with preservation of (i'). 
Again by density of $P_{N}\left({ }^{m+n} E^{\prime}\right) \otimes F$ in $P_{N}\left({ }^{m+n} E^{\prime} ; F\right)$, to prove (ii') it is enough to consider $\vec{Q}_{m+n}$ of the form $\vec{Q}_{m+n}=Q_{m+n} \cdot y, Q_{m+n} \in P_{N}\left({ }^{m+n} E^{\prime}\right)$, $y \in F$ : using the reduction from $\langle,\rangle_{m, F}$ to $\langle,\rangle_{m}$ (and likewise for $\langle,\rangle_{m+n, F}$ ) from Proposition I.2, (ii') follows then from the identity of Proposition III.1(ii). $\square$

We extend $P_{n}^{\prime}(d)_{F}$ to $P_{N}\left(E^{\prime} ; F\right)$ by linearity, i.e.:

for $\vec{P}_{k} \in P_{N}\left({ }^{k} E^{\prime} ; F\right)$.

$$
P_{n}^{\prime}(d)_{F}\left(\sum_{k=0}^{M} \vec{P}_{k}\right):=\left\{\begin{array}{l}
\sum_{k=n}^{M} P_{n}^{\prime}(d)_{F} \vec{P}_{k} \quad \text { if } M \geqslant n, \\
0 \quad \text { if } M<n,
\end{array}\right.
$$

Proposition III.2. $P_{n}^{\prime}(d)_{F}$ can be uniquely extended as a continuous linear operator on $\mathrm{H}_{N b}\left(E^{\prime} ; F\right)$, such that

$$
\left\|P_{n}^{\prime}(d)_{F} \vec{f}\right\|_{N, \rho} \leqslant n ! \rho^{-n}\left\|P_{n}^{\prime}\right\|\|\vec{f}\|_{N, 2 \rho},
$$

for every $\rho>0$ and $\vec{f} \in H_{N b}\left(E^{\prime} ; F\right)$.

Proof. By the density of $P_{N}\left(E^{\prime} ; F\right)$ in $H_{N b}\left(E^{\prime} ; F\right)$ and the fact that the linear extension of $P_{n}^{\prime}(d)_{F}$ maps $P_{N}\left(E^{\prime} ; F\right)$ into itself, it is enough to consider $\vec{f}=$ $\left.\Sigma_{k=0}^{M} \vec{Q}_{k}, \vec{Q}_{k} \in P_{N}{ }^{k} E^{\prime} ; F\right)$ : then $P_{n}^{\prime}(d)_{F} \vec{f}:=\Sigma_{k=n}^{M} P_{n}^{\prime}(d)_{F}=\sum_{m=0}^{M-n} P_{n}^{\prime}(d)_{F} \vec{Q}_{m+n}$ (the case $M<n$ being trivial), so $(1 / m !) \hat{d}^{m}\left(P_{n}^{\prime}(d)_{F} \vec{f}\right)(0)=P_{n}^{\prime}(d)_{F} \vec{P}_{m+n}$. By use of the estimate of Proposition II.1' we get

$$
\begin{aligned}
\left\|P_{n}^{\prime}(d)_{F} \vec{f}\right\|_{N, \rho} & :=\sum_{m=0}^{M-n} \frac{1}{m !} \rho^{m}\left\|m ! P_{n}^{\prime}(d)_{F} \vec{Q}_{m+n}\right\|_{N} \\
& \leqslant \rho^{-n} \sum_{k=n}^{M} \frac{1}{(k-n) !} \rho^{k}\left\|P_{n}^{\prime}\right\|\left\|\vec{Q}_{m+n}\right\|_{N}\left(\begin{array}{l}
k \\
n
\end{array}\right) \\
& \leqslant \rho^{-n}\left\|P_{n}^{\prime}\right\| n ! \sum_{k=n}^{N} \frac{1}{k !} \rho^{k}\left\|\vec{Q}_{k}\right\|_{N} 2^{k} \leqslant \rho^{-n} n !\left\|P_{n}^{\prime}\right\|\|\vec{f}\|_{N, 2 \rho} .
\end{aligned}
$$

Proposition III.3. For each $g^{\prime} \in \operatorname{Exp}(E)$, with $g_{n}^{\prime}:=(1 / n !) \hat{d}^{n} g^{\prime}(0)$, and $\vec{f} \in H_{N b}\left(E^{\prime} ; F\right)$, the series $\Sigma_{n=0}^{\infty} g_{n}^{\prime}(d)_{F} \vec{f}$ converges in $H_{N b}\left(E^{\prime} ; F\right)$.

Proof. By definition of $\operatorname{Exp}(E)$ there is a $\lambda>\lim \sup _{n}\left\{n !\left\|g_{n}^{\prime}\right\|\right]^{1 / n}$, hence a $C \geqslant 1$ such that $n !\left\|g_{n}^{\prime}\right\| \leqslant C \lambda^{n}$ for all $n \in N$. Given any integer $m>0$ and any $\rho>0$, choose $\sigma>\operatorname{Max}(\lambda, \rho)$ : using the estimates \|\|$_{\rho} \leqslant\|\|_{\sigma}$ and those on $g_{n}^{\prime}(d)$ obtained in Proposition III.2 we get, for every $\vec{f} \in H_{N b}\left(E^{\prime} ; F\right)$ :

$$
\begin{aligned}
\left\|\sum_{n=0}^{M} g_{n}^{\prime}(d)_{F} \vec{f}\right\|_{N, \rho} & \leqslant \sum_{n=0}^{M}\left\|g_{n}^{\prime}(d)_{F} \vec{f}\right\|_{N, \sigma} \leqslant\|\vec{f}\|_{N, 2 \sigma} \sum_{n=0}^{M} n ! \sigma^{n}\left\|g_{n}^{\prime}\right\| \\
& \leqslant\|\vec{f}\|_{N, 2 \sigma} C \sum_{n=0}^{M}\left(\frac{\lambda}{\sigma}\right)^{n} \leqslant C\left(1-\frac{\lambda}{\sigma}\right)^{-1}\|\vec{f}\|_{N, 2 \sigma} ;
\end{aligned}
$$


convergence as $M \rightarrow \infty$ follows by the completeness of $H_{N b}\left(E^{\prime} ; F\right)[\mathrm{G} 2, \S 5$, Proposition 2, p. 43].

Definition. Given $g^{\prime} \in \operatorname{Exp}(E)$ and letting $g_{n}^{\prime}:=(1 / n !) \hat{d}^{n} g(0)$ we define the differential operator of (possibly) infinite order of scalar type $g^{\prime}(d)_{F}$ on $\vec{f} \in$ $H_{N b}\left(E^{\prime} ; F\right)$ by $g^{\prime}(d)_{F} \vec{f}:=\Sigma_{n=0}^{\infty} g_{n}^{\prime}(d)_{F} \vec{f}$. From the estimate in the proof of Proposition III.3 we have immediately:

CoROllaRY. For each $g^{\prime} \in \operatorname{Exp}(E)$, the operator $g^{\prime}(d)_{F}$ is continuous on $H_{N b}\left(E^{\prime} ; F\right)$.

REMARK. When $\operatorname{dim} F=1$ we have the differential operators of (possibly) infinite order $g^{\prime}(d)$ on $H_{N b}\left(E^{\prime}\right)$, studied in [G1] (or [G2, §7]) in the form of convolution operators (see ahead). From the density of $H_{N b}\left(E^{\prime}\right) \otimes F$ in $H_{N b}\left(E^{\prime} ; F\right)$ (Proposition II.1) it follows that $g^{\prime}(d)_{F}$ is the extension to $H_{N b}\left(E^{\prime} ; F\right)$ of the operator $g^{\prime}(d) \otimes 1_{F}$ (cf. the definition of $P_{n}^{\prime}(d)_{F}$ ).

RELATION With CONVolutions. In [G1] (or [G2, §7]) it is shown that the $g^{\prime}(d)$ are the convolution operators on $H_{N b}\left(E^{\prime}\right)$, i.e., of the form $f \mapsto T * f$ for $T \in H_{N b}\left(E^{\prime}\right)^{\prime}$, where $(T * f)\left(x^{\prime}\right):=\left\langle\tau_{-x}, f, T\right\rangle$. (It is enough to let $g^{\prime}$ be the Fourier-Borel transform $B T$ of $T$, and use the fact that $\hat{d}^{n}\left(\tau_{-x}, f\right)(0)=$ $\hat{d}^{n} f\left(x^{\prime}\right)$, together with the scalar form of the representation $\left\langle, g^{\prime}\right\rangle$ of $T$ in Proposition II.3.) It follows immediately that $g^{\prime}(d)$ commutes with translations, and in fact that all continuous linear operators $L$ on $\mathrm{H}_{N b}\left(E^{\prime}\right)$ commuting with translations are of the form $g^{\prime}(d)$ for some $g^{\prime} \in \operatorname{Exp}(E)$ (let $T(f):=(L f)(0)$ for each $f \in H_{N b}\left(E^{\prime}\right)$ and $\left.g^{\prime}:=B T\right)$. From the coincidence of $g^{\prime}(d)_{F}$ with $g^{\prime}(d) \otimes 1_{F}$ on the dense subspace $H_{N b}\left(E^{\prime}\right) \otimes F$ of $H_{N b}\left(E^{\prime} ; F\right)$ it follows that $g^{\prime}(d)_{F}$ also commutes with translations on $H_{N b}\left(E^{\prime} ; F\right)$.

As in the scalar case, any continuous linear operator $L$ on $\mathrm{H}_{N b}\left(E^{\prime} ; F\right)$ commuting with translations is of the form $\vec{f} \mapsto \vec{T} * \vec{f}$ for some $\vec{T} \in L\left(H_{N b}\left(E^{\prime} ; F\right) ; F\right)$, where $(\vec{T} * \vec{f})\left(x^{\prime}\right)=\vec{T}\left(\tau_{-x}, \vec{f}\right)$ : indeed, let $\vec{T}(\vec{f}):=(L \vec{f})(0)$ for each $\vec{f} \in$ $H_{N b}\left(E^{\prime} ; F\right)(\vec{T}$ being continuous because the evaluation map $\vec{f} \mapsto \vec{f}(0)$, as well as $L$, is continuous: $\|\vec{f}(0)\| \leqslant\|\vec{f}\|_{N, \rho}$ for any $\left.\rho\right)$. The operators $g^{\prime}(d)_{F}$ with $g^{\prime} \in$ $\operatorname{Exp}(E)$ are then the convolution operators $\vec{T} *$ with $\vec{T}=T \otimes 1_{F}$, where $T \in$ $H_{N b}\left(E^{\prime}\right)^{\prime}$ is the inverse Fourier-Borel transform of $g^{\prime}$. (We omit details since we will not need this representation.)

DUALITY OF DIFFERENTIAL AND MULTIPLICATION OPERATORS. In [G1] (or [G2, §7]) it is shown that the Fourier-Borel transformation $B$ is an algebra isomorphism from $H_{N b}\left(E^{\prime}\right)^{\prime}$ with convolution (where $\left(T_{1} * T_{2}\right)(f):=T_{1} *$ $\left(T_{2} * f\right)(0)$ for $T_{1}, T_{2} \in H_{N b}\left(E^{\prime}\right)^{\prime}$ and $\left.f \in H_{N b}\left(E^{\prime}\right)\right)$, onto $\operatorname{Exp}(E)$ with pointwise multiplication. In other words, the transpose of $g^{\prime}(d)$ (i.e., $T *$ with $B T=$ $\left.g^{\prime}\right)$ with respect to the pairing $《, \|$ is $g^{\prime} \cdot$ (pointwise product by $g^{\prime}$ ):

Proposition III.4. $\left\langle\left\langle g^{\prime}(d) f, f^{\prime}\right\rangle\right\rangle=\left\langle\left\langle f, g^{\prime} \cdot f^{\prime}\right\rangle\right.$ for all $f \in H_{N b}\left(E^{\prime}\right)$ and $f^{\prime}$, $g^{\prime} \in \operatorname{Exp}(E)$. 
Proof. [G2, §7, Proposition 2, p. 60].

The same holds in the vector-valued case, for operators of scalar type:

PROPOSITION III.4'. $\left\langle\left\langle g^{\prime}(d)_{F} \vec{f}, \vec{f}^{\prime}\right\rangle_{F}=\left\langle\left\langle\vec{f}, g^{\prime} \cdot \vec{f}^{\prime}\right\rangle_{F}\right.\right.$ for all $\vec{f} \in H_{N b}\left(E^{\prime} ; F\right)$, $\vec{f}^{\prime} \in \operatorname{Exp}\left(E ; F^{\prime}\right)$ and $g^{\prime} \in \operatorname{Exp}(E)$.

Proof. From the density of $H_{N b}\left(E^{\prime}\right) \otimes F$ in $H_{N b}\left(E^{\prime} ; F\right)$ (Proposition II.1) it is enough to consider $\vec{f}$ of the form $\vec{f}=f \cdot y$ with $f \in H_{N b}\left(E^{\prime}\right)$ and $y \in F$. By use of the reduction to 《, , $\rangle$ of Proposition II.4 we use Proposition III.4 to get:

$$
\begin{aligned}
\left\langle\left\langle g^{\prime}(d)_{F} \vec{f}, \vec{f}^{\prime}\right\rangle_{F}\right. & =\left\langle\left\langle g^{\prime}(d) f \cdot y, \vec{f}^{\prime}\right\rangle\right\rangle_{F}=\left\langle\left\langle g^{\prime}(d) f, \vec{f}_{y}^{\prime}\right\rangle\right\rangle \\
& =\left\langle\left\langle f, g^{\prime} \cdot \vec{f}_{y}^{\prime}\right\rangle\right\rangle=\left\langle\left\langle f,\left(g^{\prime} \cdot \vec{f}^{\prime}\right)_{y}\right\rangle\right. \\
& =\left\langle\left\langle f \cdot y, g^{\prime} \cdot \vec{f}^{\prime}\right\rangle_{F}=\left\langle\left\langle\vec{f}, g^{\prime} \cdot \vec{f}^{\prime}\right\rangle_{F} .\right.\right.
\end{aligned}
$$

Remark. Proposition III. $4^{\prime}$ can also be proved directly: it is enough to expand $\left\langle\langle,\rangle_{F}\right.$ as an infinite series in terms of the forms $\langle,\rangle_{n, F}$ given in Proposition II.3, and then to apply the identity of Proposition III.1'(ii) term by term, setting $\vec{Q}_{m+n}=\hat{d}^{m+n} f(0)$ and $Q_{m}^{\prime}=\hat{d}^{m} f^{\prime}(0)$. In fact, Proposition III.4, itself can be obtained from the scalar form of Proposition II.3 together with Proposition III.1(ii) in the same way.

\section{Chapter IV. Existence AND Approximation Theorems}

The existence and approximation theorems for convolution equations in the scalar-valued case depend on III.4 (i.e., [G2, §7, Proposition 2]), and the following "division theorem":

Proposition IV.1. Given $f \in \operatorname{Exp}(E)$ and $g^{\prime} \neq 0$ in $\operatorname{Exp}(E)$, suppose the following holds:

(*) For all $f \in$ Ker $g^{\prime}(d)$ we have $\left\langle\left\langle f, f^{\prime}\right\rangle\right\rangle=0$.

Then $f^{\prime}$ has the form $f^{\prime}=g^{\prime} \cdot h^{\prime}$ with $h^{\prime} \in \operatorname{Exp}(E)$. In fact, it is enough to suppose the functions $f$ in (*) to be of the form $f=e^{x} \cdot P$, with $P \in P_{N}\left(E^{\prime}\right)$ and $x \in E$.

Proof. This is Proposition 3, $\S 8$, of [G2] (done there over $E^{\prime}$ ), in the language of convolutions: one shows that (*) implies $f^{\prime} / g^{\prime}$ is analytic on all affine lines $S$ in $E$ where $g^{\prime}$ is not identically zero, by showing that $(*)$ implies that every zero of $\left.g^{\prime}\right|_{S}$ is also a zero of no smaller order of $\left.f^{\prime}\right|_{S}$. One then shows that the analyticity of these $f^{\prime} /\left.g^{\prime}\right|_{S}$ implies that $f^{\prime} / g^{\prime}$ has an entire extension $h^{\prime} \in$ $H(E)$ by an integral formula [G2, §8, Proposition 2, p. 65]. One then shows that a quotient of entire functions of exponential type on a Banach space is again of exponential type, by means of a one-dimensional growth estimate of Malgrange's [G2, §8, Proposition 1, p. 63].

We now extend this fundamental division theorem to the vector-valued situation. 
Proposition IV.1'. Given $g^{\prime} \neq 0$ in $\operatorname{Exp}(E)$ and $\vec{f}^{\prime} \in \operatorname{Exp}\left(E ; F^{\prime}\right)$, suppose the following holds:

$\left(*^{\prime}\right)$ For all $\vec{f} \in \operatorname{Ker} g^{\prime}(d)_{F}$ we have $\left.\left\langle\vec{f}, \vec{f}^{\prime}\right\rangle\right\rangle_{F}=0$. Then $\vec{f}^{\prime}$ has the form $\vec{f}^{\prime}=g^{\prime} \cdot \vec{h}^{\prime}$ with $\vec{h}^{\prime} \in \operatorname{Exp}\left(E ; F^{\prime}\right)$. In fact, it is enough to suppose the functions $\vec{f}$ in $\left(*^{\prime}\right)$ to be of the form $\vec{f}=e^{x} \cdot \vec{P}$, with $\vec{P} \in P_{N}\left(E^{\prime} ; F\right)$ and $x \in E$.

The proof requires two lemmas:

LEMMA IV.1. If $\left(*^{\prime}\right)$ holds then the following also holds:

$\left(*^{\prime \prime}\right)$ for all $f \in \operatorname{Ker} g^{\prime}(d)$ and $y \in F$ we have $\left\langle\left\langle f, \vec{f}_{y}^{\prime}\right\rangle=0\right.$.

If the functions $\vec{f}$ in $\left(*^{\prime}\right)$ are required only to be of the form $\vec{f}=e^{x} \cdot \vec{P}, \vec{P} \in$ $P_{N}\left(E^{\prime} ; F\right), x \in E$, then the functions $f$ in $\left(*^{\prime \prime}\right)$ need only be of the form $f=$ $e^{x} \cdot P, P \in P_{N}\left(E^{\prime}\right), x \in E$.

Proof. Suppose $\left(*^{\prime \prime}\right)$ failed: then there would be some $f \in \operatorname{Ker} g^{\prime}(d)$ and $y \in F$ such that $\left\langle f, \vec{f}_{y}^{\prime}\right\rangle \neq 0$. But then from the "reduction from $\langle,\rangle_{F}$ to $\langle$,$\rangle "$ of Proposition II.4 we get $\left\langle\left\langle f \cdot y, \vec{f}^{\prime}\right\rangle_{F} \neq 0\right.$. By setting $\vec{f}:=f \cdot y$ in $\left(*^{\prime}\right)$ we get a contradiction. Moreover, if $f$ were of the form $f=e^{x} \cdot P, P \in P_{N}\left(E^{\prime}\right), x \in E$, then $\vec{f}$ would be of the form $\vec{f}=e^{x} \cdot \vec{P}$, with $\vec{P}:=P \cdot y \in P_{N}\left(E^{\prime} ; F\right)$, so we would still have a contradiction in the alternate form of $\left(*^{\prime}\right)$.

Lemma IV.2. Given $g^{\prime} \neq 0$ in $\operatorname{Exp}(E)$ and $\vec{h}^{\prime} \in H\left(E^{\prime} ; F\right)$, if $g^{\prime} \cdot \vec{h}^{\prime} \in$ $\operatorname{Exp}\left(E ; F^{\prime}\right)$ then also $\vec{h}^{\prime} \in \operatorname{Exp}\left(E ; F^{\prime}\right)$.

Proof. Malgrange's estimate on quotients of functions in $\operatorname{Exp}(C)$ is shown in [G2, §8, Proposition 1 and Lemma 1, p. 64], to lead to an estimate of the form $\left|f_{3}^{\prime}(x)\right| \leqslant C_{3} e^{c_{3}\|x\|}$ for constants $C_{3} \geqslant 0$ and $c_{3} \geqslant 0$ and any $x \in E$, whenever $f_{3}^{\prime} \in H(E)$ and $f_{2}^{\prime} \cdot f_{3}^{\prime}=f_{1}^{\prime}$, for $f_{1}^{\prime}, f_{2}^{\prime}$ satisfying inequalities of the same form, i.e.,

$$
\left.\left|f_{i}^{\prime}(x)\right| \leqslant C_{i} e^{c_{i}\|x\|} \quad \text { (i.e., for } f_{i}^{\prime} \in \operatorname{Exp}(E)\right), i=1,2 .
$$

Since $g^{\prime} \cdot \vec{h}^{\prime} \in \operatorname{Exp}\left(E ; F^{\prime}\right)$ by hypothesis, it follows that $g^{\prime} \cdot \vec{h}^{\prime}$ satisfies an estimate of the form $\left\|g^{\prime} \cdot \vec{h}^{\prime}(x)\right\| \leqslant C_{1} e^{c_{1}\|x\|}$ for every $x \in E$; hence for every $y \in F$ with $\|y\|=1$ we have $\left|g^{\prime} \cdot \vec{h}_{y}^{\prime}(x)\right| \leqslant C_{1} e^{c_{1}\|x\|}$, where $C_{1}$ and $c_{1}$ are independent of $y$. Moreover, $g^{\prime} \in \operatorname{Exp}(E)$ implies that $g^{\prime}$ satisfies an estimate of the form $\left|g^{\prime}(x)\right| \leqslant C_{2} e^{c_{2}\|x\|}$ for all $x$. Letting $f_{1}^{\prime}:=g^{\prime} \cdot \vec{h}_{y}^{\prime}$ and $f_{2}^{\prime}:=g^{\prime}$, from the Malgrange-Gupta estimate we obtain $\left|\vec{h}_{y}^{\prime}(x)\right| \leqslant C_{3} e^{c_{3}\|x\|}$ for all $x \in E$ and all $y \in F$ with $\|y\|=1$, since $C_{3}, c_{3}$ depend only on the $C_{i}, c_{i}, i=1,2$, while these last constants are independent of $y,\|y\|=1$. But then we have $\left\|\vec{h}^{\prime}(x)\right\| \leqslant C_{3} e^{c_{3}\|x\|}$ for all $x \in E$, i.e., $\vec{h}^{\prime} \in \operatorname{Exp}\left(E ; F^{\prime}\right)$.

Proof of Proposition IV.1'. Given (*'), from the first lemma it follows that $(*)$ in Proposition IV.1 holds for $f^{\prime}:=\vec{f}_{y}^{\prime}$, for each $y \in F$. Hence by the 
conclusion of Proposition IV.1 it follows that, for every such $y$ there exists an $h_{(y)}^{\prime} \in \operatorname{Exp}(E) \subset H(E)$ such that $\vec{f}_{y}^{\prime}=g^{\prime} \cdot h_{(y)}^{\prime}$. We define $\vec{h}^{\prime}: E \rightarrow F^{*}$ by

$$
\begin{aligned}
& \left\langle y, \vec{h}^{\prime}(x)\right\rangle:=h_{(y)}^{\prime}(x) \text { for each } x \in E \text { and } y \in F: \\
& \text { then } \vec{f}_{y}^{\prime}=g^{\prime} \cdot \vec{h}_{y}^{\prime}=\left(g^{\prime} \cdot \vec{h}^{\prime}\right)_{y} \text { for every } y \in F \text {, i.e., } \vec{f}^{\prime}=g^{\prime} \cdot \vec{h}^{\prime} .
\end{aligned}
$$

$\vec{h}^{\prime}(E) \subset F^{\prime}$ : indeed, given any $x \in E$, since $g^{\prime}$ is not identically zero it follows from a well-known result on convergent power series, valid also in infinitedimensional domains [H, §III.1.3, Theorem 3(b), p. 71], that $g^{\prime}$ cannot vanish on any neighborhood of $x$. Hence there is a sequence of $x_{n} \in E$ such that $x_{n} \rightarrow x$ in $E$ and $g^{\prime}\left(x_{n}\right) \neq 0$ for every $n$. Hence $\vec{h}^{\prime}\left(x_{n}\right)=g^{\prime}\left(x_{n}\right)^{-1} \vec{f}^{\prime}\left(x_{n}\right) \in F^{\prime}$ for each $n$, and moreover

$$
\left\langle y, \vec{h}^{\prime}\left(x_{n}\right)\right\rangle:=h_{(y)}^{\prime}\left(x_{n}\right) \rightarrow \vec{h}_{(y)}^{\prime}(x)=:\left\langle y, \vec{h}^{\prime}(x)\right\rangle
$$

as $n \rightarrow \infty$ for every $y \in F$ by continuity of the functions $h_{(y)}^{\prime}$. It follows that $\vec{h}^{\prime}(x) \in F^{\prime}$ by the uniform boundedness principle.

$\vec{h}^{\prime}$ is $G$-analytic, i.e., analytic along all complex affine lines in $E$ : indeed, each $\langle y,\rangle \circ \vec{h}^{\prime}=h_{(y)}^{\prime}$ is analytic, and the $\langle y,\rangle \in F^{\prime \prime}$ for all $y \in F$ form a determining space of continuous linear forms on $F^{\prime}$, so the $G$-analyticity of $\vec{h}^{\prime}$ follows from a classic result [Dun, p. 354].

$\vec{h}^{\prime}$ is also locally bounded: indeed, it is enough to show that $\vec{h}^{\prime}(K)$ is bounded for every compact set $K$ in $E$. From the coincidence of weakly and strongly bounded sets in $F^{\prime}$ [H, §III.1.1, Proposition 1(b), p. 56] it is then enough to show that $\left(y^{\prime \prime} \circ \vec{h}^{\prime}\right)(K)$ is bounded for each $y^{\prime \prime} \in F^{\prime \prime}$. But from the compactness of $K$ and the continuity of each $h_{(y)}^{\prime}$ it follows that $\left\{\left\langle y, \vec{h}^{\prime}(k)\right\rangle \mid k \in K\right\}$ is bounded by some $C_{y}>0$, for every $y \in F$. But by Alaoglu's theorem, $F$ is weak*-densely imbedded in $F^{\prime \prime}$. Hence for each $y^{\prime \prime} \in F^{\prime \prime}$ we can find some $y \in F$ such that $\left|\left\langle y^{\prime}, y^{\prime \prime}\right\rangle-\left\langle y, y^{\prime}\right\rangle\right| \leqslant 1$ for every $y^{\prime} \in F^{\prime}$, so in particular $\left|\left\langle\vec{h}^{\prime}(k), y^{\prime \prime}\right\rangle\right| \leqslant 1+$ $\left|\left\langle y, \vec{h}^{\prime}(k)\right\rangle\right| \leqslant 1+C_{y}$ for every $k \in K$, i.e., each $\left(y^{\prime \prime} \circ \vec{h}^{\prime}\right)(K)$ is bounded.

The $G$-analyticity and local boundedness of $\vec{h}^{\prime}$ imply that $\vec{h}^{\prime} \in H\left(E ; F^{\prime}\right)[H$, $\S$ III.2.2, Proposition 1]. Finally from the second lemma above and the exponential nature of $\vec{f}^{\prime}$ and $g^{\prime}$ we conclude that $\vec{h}^{\prime} \in \operatorname{Exp}\left(E ; F^{\prime}\right)$.

We can now state and prove the Malgrange-Gupta existence and approximation theorems for $g^{\prime}(d)_{F}$. The proofs, given here in terms of the form $\left.\|,\right\rangle_{F}$, are analogous to those in the scalar-valued case:

THEOREM IV.1. Given $g^{\prime} \neq 0$ in $\operatorname{Exp}(E)$, every equation $g^{\prime}(d)_{F} \vec{f}=\vec{g}$ with $\vec{g}$ in $H_{N b}\left(E^{\prime} ; F\right)$ has a solution $\vec{f}$ in $H_{N b}\left(E^{\prime} ; F\right)$.

Proof. The operator $g^{\prime} \cdot\left(\right.$ multiplication by $g^{\prime}$ on $\operatorname{Exp}\left(E ; F^{\prime}\right)$ ) is the adjoint of $g^{\prime}(d)_{F}$ with respect to the pairing given by 《 , $\rangle_{F}$ (Proposition III.4'). Moreover, $g^{\prime} \bullet$ is injective, as follows easily from the fact that if $g^{\prime} \neq 0$ and $g^{\prime}$. 
$\vec{h}^{\prime}=0$ then $\vec{h}^{\prime}$ vanishes on a neighborhood of a point where $g^{\prime}$ is not zero. Besides, $\operatorname{Im}\left(g^{\prime} \cdot\right)\left(:=\left\{g^{\prime} \cdot \vec{h}^{\prime} \mid \vec{h}^{\prime} \in \operatorname{Exp}\left(E ; F^{\prime}\right)\right\}\right)$ is closed with respect to the weak topology of the pairing $\|,\|_{F}$ : indeed, from the $\left.\|,\right\rangle_{F}$-duality of $g^{\prime}(d)_{F}$ and $g^{\prime} \cdot$ it follows easily that $\operatorname{Im}\left(g^{\prime} \cdot\right) \subset \operatorname{Ker}\langle\vec{f},\rangle_{F}$ for every $\vec{f} \in \operatorname{Ker} g^{\prime}(d)_{F}$. Conversely, from Proposition IV.1' it follows that $\operatorname{Ker}\langle\vec{f},\rangle_{F} \subset \operatorname{Im}\left(g^{\prime} \cdot\right)$ for all $\vec{f} \in \operatorname{Ker} g^{\prime}(d)_{F}$. Hence $\operatorname{Im}\left(g^{\prime} \cdot\right)$ is an intersection of weakly closed sets. Finally, from the Dieudonne-Schwartz theorem on surjections on Fréchet spaces [ $\mathbf{T r}$, Theorem 37.2, p. 383] it follows that $g^{\prime}(d)_{F}$ is surjective.

THEOREM IV.2. Given $g^{\prime} \in \operatorname{Exp}(E)$, the solutions of $g^{\prime}(d)_{F} \vec{f}=0$ of the form $\vec{f}=e^{x} \cdot \vec{P}$ with $\vec{P} \in P_{N}\left(E^{\prime} ; F\right)$ and $x \in E$ are total in the space of all solutions in $H_{N b}\left(E^{\prime} ; F\right)$.

Proof. If $g^{\prime}=0$ then $\operatorname{Ker} g^{\prime}(d)_{F}=H_{N b}\left(E^{\prime} ; F\right)$, so we are reduced to the density in $H_{N b}\left(E^{\prime} ; F\right)$ of the subspace generated by the functions $e^{x} \cdot y, y \in F$, $x \in E$. [G2, §5, Proposition 3]. Let then $g^{\prime} \neq 0$ : given $\left.\left.\|, \vec{f}^{\prime}\right\rangle\right\rangle_{F}$ in $H_{N b}\left(E^{\prime} ; F\right)^{\prime}$ suppose $\left\langle\left\langle e^{x} \cdot \vec{P}, \vec{f}^{\prime}\right\rangle\right\rangle_{F}=0$ for all $\vec{P} \in P_{N}\left(E^{\prime} ; F\right)$ and $x \in E$ : by Proposition IV.1', $\vec{f}^{\prime}$ has then the form $\vec{f}^{\prime}=g^{\prime} \cdot \vec{h}^{\prime}$ for some $\vec{h}^{\prime} \in \operatorname{Exp}\left(E ; F^{\prime}\right)$. By Proposition III.4' it follows that $\left\langle\left\langle\vec{f}, \vec{f}^{\prime}\right\rangle_{F}=\left\langle\vec{f}, g^{\prime} \cdot \vec{h}^{\prime}\right\rangle_{F}=\left\langle\left\langle g^{\prime}(d)_{F} \vec{f}, \vec{h}^{\prime}\right\rangle\right\rangle_{F}=0\right.$ for every $\vec{f} \in$ $\operatorname{Ker} g^{\prime}(d)_{F}$. Since every $T \in H_{N b}\left(E^{\prime} ; F\right)^{\prime}$ is of the form $\left.\|, \vec{f}^{\prime}\right\rangle_{F}$ (where $B T=$ $\left.\vec{f}^{\prime}\right)$ (Proposition II.2), it follows that all $T \in H_{N b}\left(E^{\prime} ; F\right)^{\prime}$ which are zero on the solutions $e^{x} \cdot \vec{P}$ are also zero on the space of all solutions $\vec{f} \in H_{N b}\left(E^{\prime} ; F\right)$ of $g^{\prime}(d)_{F} \vec{f}=0$. The Hahn-Banach theorem then implies the density of the subspace generated by the solutions $e^{x} \cdot \vec{P}$.

\section{REFERENCES}

[A]. R. Aron, Tensor products of holomorphic functions, Nederl. Akad. Wetensch. Proc. Ser. A 76 = Indag. Math 35 (1973), $192-202$.

[A-B]. R. Aron and Ph. Boland, Unpublished results.

[B1]. Ph. Boland, Espaces pondérés de fonctions entières et de fonctions entieres nucléaires dans les espaces vectoriels topologiques réels ou complexes, $\mathrm{C}$. R. Acad. Sci. Paris Sér. A-B 270 (1970), A643-A646.

[B2]. - Some spaces of entire and nuclearly entire functions on a Banach space, J. Reine Angew. Math., Part II, 271 (1974), 8-27.

[B3]. - Malgrange theorem for entire functions on nuclear spaces, Proc. on Infinite-Dimensional Holomorphy, Lecture Notes in Math., vol. 364, Springer-Verlag, New York, 1974.

[B-D]. Ph. Boland and S. Dineen, Convolution operators on G-holomorphic functions in infinite dimensions, Trans. Amer. Math. Soc. 190 (1974), 313-323.

[Di]. S. Dineen, Holomorphy types on a Banach space, Studia Math. 39 (1971), 241 288. MR 46 \#3837.

[D1]. T. A. W. Dwyer III, Partial differential equations in Fischer-Fock spaces for the Hilbert-Schmidt holomorphy type, Bull. Amer. Math. Soc. 77 (1971), 725-730. MR 44 \#7288.

[D2]. Holomorphic Fock representations and partial differential equations on countably Hilbert spaces, Bull. Amer. Math. Soc. 79 (1973), 1045-1050. MR 47 \#9283. 
[D3]. - Partial differential equations in holomorphic Fock spaces, Proc.

Sympos. Anal. (Recife, July, 1972), L. Nachbin, (Editor), Lecture Notes in Math., vol. 364, Springer-Verlag, New York, 1974.

[D4]. - Holomorphic representation of tempered distributions and weighted Fock spaces, Proc. Colloq. Analyse (Rio de Janeiro, August, 1972), L. Nachbin, (Editor), Actualités Sci. Indust., no. 1367, Hermann, Paris, 1975, pp. 95, 118.

[D5]. - Dualité des espaces de fonctions entières en dimension infinie, C. R. Acad. Sci. Paris Ser A-B 281 (1975), A1439-A1442.

[D6]. - Équations différentielles d'ordre infini dans des espaces localement convexes, C. R. Acad. Sci. Paris Ser. A-B 281 (1975), A163-A166. $305-356$.

[Dun]. N. Dunford, Uniformity in linear spaces, Trans. Amer. Math. Soc. 44 (1938),

[Gr]. A. Grothendieck, Sur certains espaces de fonctions holomorphes. II, J. Reine Angew. Math. 192 (1953), 77-95. MR 15, 963.

[G1]. C. Gupta, On the Malgrange theorem for nuclearly entire functions of bounded type on a Banach space, Notas de Matemática, no. 37, I. M. P. A., Rio de Janeiro, Brasil, 1968.

[G2]. Convolution operators and holomorphic mappings on a Banach space, Sem. Anal. Mod. No. 2, Univ. Sherbrooke, Québec, 1969.

[G3]. - On the Malgrange theorem for nuclearly entire functions of bounded type on a Banach space, Nederl. Akad. Wetensch. Proc. Ser. A 73 = Indag Math 32 (1970), 356-358. MR 44 \#7289.

[H]. M. Hervé, Analytic and plurisubharmonic functions in finite and infinite dimensions, Lecture Notes in Math., vol. 198, Springer-Verlag, New York, 1970.

[Mat]. M. C. Matos, Sur le théorème d'approximation et d'existence de Malgrange Gupta, C. R. Acad. Sci. Paris Sér. A-B 271 (1970), A1258-A1259. MR 44 \#3105.

[N1]. L. Nachbin, Topology on spaces of holomorphic mappings, Ergebnisse der Mathematik und ihrer Grenzgebiete, Band 47, Springer-Verlag, New York 1969. MR 40 \#7787.

[N2]. Convolution operators in spaces of nuclearly entire functions on a Banach space, Functional analysis and related fields (Chicago 1968), Springer-Verlag, Berlin, 1970, pp. 167-171.

[P]. D. Pisanelli, Sull'integrazione di un sistema di equazioni ai differenziali totali in uno spazio di Banach, Atti Accad. Naz. Lincei Rend. Cl. Sci. Fis. Mat. Natur. (8) 46 (1969), 672-681. MR 41 \#8991.

[Tr]. F. Trèves, Topological vector spaces, distributions and kernels, Academic Press, New York, 1967. MR 37 \#726.

DEPARTMENT OF MATHEMATICAL SCIENCES, NORTHERN ILLINOIS UNIVERSITY, DEKALB, ILLINOIS 60115 